\title{
Percutaneous coronary intervention 2005
}

$\mathrm{t}$ is a pleasure for me to introduce this Heart supplement, commissioned by the British Cardiovascular Intervention Society (BCIS), and generously funded by the BCIS industry partner, the British Cardiovascular Industry Association (BCIA).

Under the guidance of BCIS, developments in percutaneous coronary intervention (PCI) in the UK are progressing at an exciting rate. Increasing PCI activity with an annual growth rate of $15-20 \%$ has been coupled with the enthusiastic uptake of new technology. As I write, the use of drug eluting stents in the UK is one of the highest in Europe, despite the funding constraints inherent in the National Health Service. Bringing down hospital waiting lists may be politically motivated, but there is no doubt that the impressive increase in NHS funding has benefited the cardiology community, both in terms of capital investment and the revenue stream for increased activity.

Nevertheless, there are areas in interventional cardiology that remain contentious, technically difficult, or scientifically under investigated, and we have aimed to address some of these topics in this supplement.

There is an overwhelming evidence base in support of primary PCI for ST segment elevation myocardial infarction; Simon Dixon explores novel techniques for myocardial preservation, which have major implications for the long term outcome in this group of patients. Optimal population based management of the infarct patient remains contentious; Peter Schofield addresses the issue of pre-hospital thrombolysis, and Henning Anderson and colleagues make the case for myocardial infarction centres. The role of antiplatelet treatment in optimising the haematological milieu of the PCI patient has been recognised for over 30 years; the available drugs continue to evolve and Robert Kelly and Steven Steinhubl have brought us up to date with the contemporary therapeutic options. "Stent mania" has overtaken the interventional community; Richard Lowe and colleagues address the issue of stent coating, while Tony Gershlick provides an update on drug elution. Technology comes and goes, perhaps none more so than intracoronary brachytherapy; Marytn Thomas makes the case for the ongoing application of brachytherapy for the treatment of diffuse in-stent restenosis. Treatment of the left main lesion remains on the cusp of PCI and surgical revascularisation; Kunihiko Kosuga and Hideo Tamai have explored this controversy in a case report from Japan, and Nick Curzen emphasises the move to treatment by PCI in left main patients. Finally, chronic total occlusions; Shahid Aziz and David Ramsdale report on the newer technology that may help us with this difficult group of patients.

Thank you to the authors who contributed to the supplement-high quality papers delivered in a timely fashion. Special thanks are due to the de Belder brothers, Adam and Mark, who have been dogged in their tenacity in producing the supplement, and to Stephanie Watkins of BMJ Publishing Group whose technical expertise has made the supplement possible. British Cardiovascular Intervention Society keith@dawkins.org 